\title{
SÍNDROME DE DÉFICIT DE TESTOSTERONA Y DISFUNCIÓN ERÉCTIL
}

\author{
Manuel Gil Salom? y Jose María Martínez Jabaloyas².
}

\begin{abstract}
'Servicio de Urología. Hospital Universitario Doctor Peset. Valencia. España. 'Departamento Universitario de Cirugía. Universidad de Valencia. España.

${ }^{2}$ Servicio de Urología. Hospital Clínico Universitario. Valencia. España.
\end{abstract}

Resumen.- OBJETIVO: Analizar la relación entre el síndrome de déficit de testosterona (SDT) y la disfunción eréctil y sus implicaciones diagnósticas y terapéuticas.

MÉTODO: Revisión bibliográfica mediante la base de datos de Pubmed de la US National Library of Medicine.

RESULTADOS: La real prevalencia del SDT es desconocida, debido a la falta de criterios diagnósticos uniformes en cuanto a qué fracción de testosterona debe medirse (total, libre o biodisponible) y cuáles deben ser los valores diagnósticos. A pesar de ello, se estima que entre un 5-15\% de varones con disfunción eréctil presentan niveles séricos disminuidos de testosterona. Exis-

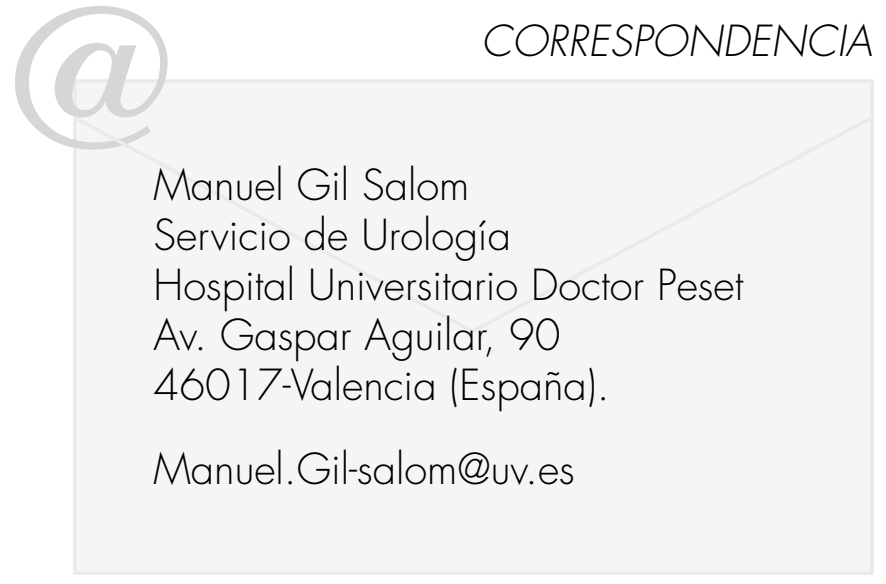

te una sólida base experimental que demuestra que la testosterona juega un papel fundamental en la fisiología de la erección, tanto a nivel central como periférico. No obstante, las evidencias obtenidas en humanos no son tan firmes, especialmente en los varones mayores con SDT. Los resultados de algunos meta-análisis demuestran que el tratamiento sustitutivo con testosterona (TST) permite mejorar las erecciones y el deseo sexual. Sin embargo, no todos los varones con SDT se benefician del TST, probablemente porque en algunos casos el origen de la disfunción eréctil es multifactorial. El tratamiento combinado con testosterona más inhibidores de fosofodiesterasa 5 (PDE5) parece ser un recurso adecuado para rescatar pacientes con disfunción eréctil e hipogonadismo que no responden a la monoterapia, ya sea ésta testosterona sóla o inhibidores de PDE5 sólos.

CONCLUSIONES: la determinación sistemática de testosterona sérica en los pacientes que consultan por disfunción eréctil es altamente recomendable, ya que el TST permite, en una proporción de pacientes, mejorar las erecciones y el deseo sexual. Además, el TST puede mejorar el resto de síntomas del SDT y aumentar la eficacia de los inhibidores de la PDE5 cuando éstos no son eficaces como monoterapia.

Palabras clave: Testosterona. Síndrome déficit testosterona. Hipogonadismo. Inhibidores fosfodiesterasa 5. Disfunción eréctil.

Summary.- OBJECTIVES: To analyze the relationship between testosterone deficit syndrome (TDS) and erectile dysfunction and its diagnostic and therapeutic implications.

METHODS: Bibliographic review in the Pub Med database of the US National Library of Medicine. 
RESULTS: The real TDS is unknown, due to the lack of uniform diagnostic criteria on what fraction should be measured (total, free or bioavailable) and what the diagnostic values are. Despite this fact, it is estimated that between $5-15 \%$ of males with erectile dysfunction show diminished testosterone levels. There is a solid research base demonstrating that testosterone plays an essential role in the physiology of erection, both at central and peripheral levels. Nevertheless, evidence obtained in human studies is not that strong, mainly in old patients with TDS. The results of some metaanalysis show that substitutive treatment with testosterone improves erections and sexual desire. However, not every patient with TDS will benefit from testosterone substitution therapy, probably because in some cases the origin of erectile dysfunction is multifactorial. Combined treatment with testosterone plus phosphodiesterase 5 (PDE 5) seems to be an adequate alternative to rescue patients with erectile dysfunction and hypogonadism not responding to monotherapy, be it with testosterone alone or PDE 5 inhibitors alone.

CONCLUSIONS: Systematic determination of serum testosterone in patients consulting for erectile dysfunction is highly recommendable, because testosterone substitution therapy enables, in a number of patients, improvement of erections and sexual desire. Moreover, testosterone substitution therapy may improve the other symptoms of TDS and increase the efficacy of PDE5 inhibitors when they are not effective in monotherapy.

Keywords: Testosterone. Testosterone deficit syndrome. Hipogonadism. Phosphodiesterase 5. Erectile dysfunction.

\section{INTRODUCCIÓN}

La disfunción eréctil (DE) y el síndrome de déficit de testosterona (SDT) son dos entidades diferentes pero que frecuentemente coexisten en los hombres de edad mediana o avanzada. Aunque cada entidad tiene sus propios mecanismos fisiopatológicos, los andrógenos desempeñan un papel fundamental en la función sexual, incluyendo la función eréctil. La testosterona es el principal andrógeno y su producción principal se localiza en el varón a nivel testicular en las células de Leydig, bajo la influencia de la hormona Luteinizante (LH) producida en la adenohipófisis.

Desde hace mucho se conoce que el hipogonadismo en el varón generalmente se acompaña de pérdida de libido y potencia sexual, que pueden ser restablecidas en ocasiones mediante administración de testosterona. Inicialmente se pensaba que la testosterona tenía una acción predominante sobre el interés sexual, siendo menos aparentes los efectos de la testosterona sobre la propia función eréctil (1). Sin embargo, recientemente se han acumulado múltiples evidencias a nivel experimental de que la testosterona ejerce también un potente efecto sobre el sustrato anatómico y fisiológico de la erección (2).

\section{Síndrome de Déficit de Testosterona}

EI SDT o Hipogonadismo de Inicio Tardío (HIT) es un síndrome clínico y bioquímico que se desarrolla con el envejecimiento y que se caracteriza por una serie de síntomas y por un déficit en los niveles séricos de testosterona, pudiendo dar lugar a alteraciones significativas de la calidad de vida y afectar adversamente la función de múltiples órganos y sistemas (3).

La sintomatología propia del SDT incluye síntomas sexuales, psíquicos y cambios en la composición corporal (Tabla I). La instauración de la sintomatología es lenta y progresiva y presenta una gran variabilidad individual (3). Se ha detectado, además, una estrecha relación entre niveles séricos bajos de testosterona y obesidad, hipertensión arterial, dislipemia, diabetes tipo 2 y el síndrome metabólico en general $(4,5)$.

Ante la inespecificidad del cuadro clínico, el diagnóstico del SDT requiere la confirmación bioquímica de los niveles séricos disminuidos de testosterona (3). La testosterona plasmática se divide en 3 fracciones (Figura 1): la fracción unida a la globulina fijadora de hormonas sexuales (SHBG), la fracción unida a la albúmina y la libre. La fracción realmente activa es la libre, que supone el $2 \%$ de la testosterona total. Debido a la lábil unión entre la testosterona y la albúmina la diferencia de la firme unión con la SHBG) existe un fácil intercambio de ésta con la libre, por lo que a estas dos fracciones (libre y unida a la albúmina) se las denomina también testosterona biodisponible (6).

La extracción sanguínea para el análisis hormonal se debe de realizar por la mañana (de 7 a 11 horas), ya que los niveles séricos de testosterona disminuyen por la tarde. La testosterona total se determina habitualmente mediante inmunoanálisis. El método de referencia para determinar la testosterona libre es la diálisis en equilibrio y para la biodisponible la precipitación con sulfato amónico, pero ambos tienen el inconveniente de ser métodos complejos y caros, por lo que son poco utilizados. De los métodos alternativos el más fidedigno es el método de cálculo matemático (a partir de los niveles de testosterona 
total, SHBG y albúmina) ya que los métodos de inmunoanálisis son muy inexactos en estos casos $(3,7,8)$. Aunque no está completamente aclarado qué fracción de la testosterona debe medirse al evaluar un cuadro de hipogonadismo, en la mayoría de estudios se ha determinado la testosterona total. Tampoco existe acuerdo sobre cuál debe ser el límite inferior de normalidad, aunque se considera que con niveles mayores a $12 \mathrm{nmol} / \mathrm{L}(350 \mathrm{ng} / \mathrm{dl})$ no se requiere tratamiento hormonal sustitutivo, mientras que los pacientes con niveles inferiores a $8 \mathrm{nmol} / \mathrm{L}$ (230 ng/dl) podrían beneficiarse del tratamiento (3). Cuando la testosterona sérica total se encuentra en niveles intermedios (entre 8 y $12 \mathrm{nmol} / \mathrm{L}$ ), se acon-

\section{TABLA I. SINTOMATOLOGÍA DEL SÍNDROME DE DÉFICIT DE TESTOSTERONA.}

\section{Síntomas Sexuales:}

- Disminución del deseo sexual

- Disminución del número y calidad de las erecciones

- Disminución del volumen del eyaculado

- Disminución de la calidad del orgasmo

- Disminución del número de erecciones nocturnas

\section{Síntomas Psíquicos:}

- Alteraciones del humor

- Disminución de la actividad intelectual

- Disminución funciones cognitivas

- Disminución orientación espacial

- Depresión

- Irritabilidad

- Insomnio

\section{Cambios en la Composición Corporal:}

- Disminución de la masa y fuerza muscular

- Aumento de la grasa visceral

- Caída del vello

- Alteraciones cutáneas

- Disminución de la densidad mineral ósea seja la determinación de testosterona libre o biodisponible, recomendándose el tratamiento con niveles de testosterona libre inferiores a $225 \mathrm{pmol} / \mathrm{ml}$ (65 $\mathrm{pg} / \mathrm{ml})(3)$.

La ausencia de unos criterios uniformes a la hora de establecer el diagnóstico condiciona que la prevalencia real del SDT sea desconocida, variando considerablemente en función de las características de la población estudiada, los valores de testosterona considerados como diagnósticos y si se ha determinado la testostrona total, libre o biodisponible. Por ejemplo, en el estudio longitudinal de Baltimore (9), se estableció como criterio de hipogonadismo una concentración sérica de testosterona total inferior a $325 \mathrm{ng} / \mathrm{dl}$ (11,3 nmol/L), detectándose así hipogonadismo en el $20 \%, 30 \%$ y $50 \%$ de varones mayores de 60, 70 y 80 años, respectivamente. Sin embargo la prevalencia de hipogonadismo observado por nosotros en varones mayores de 50 años (10) era del $4,8 \%$ si empleábamos como criterio una concentración sérica de testosterona total inferior a $250 \mathrm{ng} / \mathrm{dl}$ $(8,7 \mathrm{nmol} / \mathrm{L})$, mientras que el porcentaje ascendía hasta el $24,8 \%$ al considerar como criterio diagnóstico una concentración sérica de testosterona libre inferior a 0,228 nmol/L. Probablemente esta diferencia sea debida a la elevación de los niveles de SHBG con la edad, lo cual determina un descenso mayor de los niveles de testosterona libre que de testosterona total.

El mismo problema se plantea al intentar estimar la prevalencia de SDT entre los pacientes con disfunción eréctil. La proporción de varones con disfunción eréctil con niveles de testosterona bajos varía en la literatura entre un $1.7 \%$ y un $35 \%$ (11). En una recopilación de 9 series publicadas, Buvat y Bou Jaoudé (12) detectaron una testosterona sérica total inferior a $300 \mathrm{ng} / \mathrm{dl}$ en el $12 \%$ de un total de 7.000 pacientes con disfunción eréctil. La prevalencia de

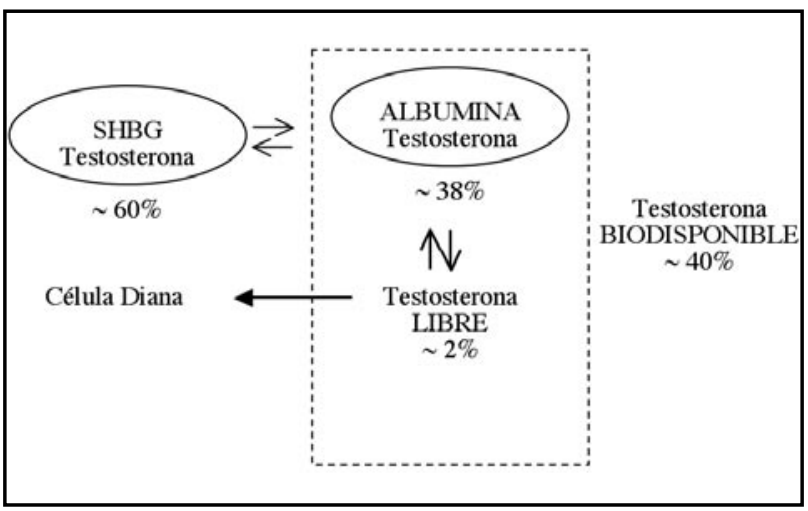

FIGURA 1. Fracciones de testosterona plasmática (SHBG: globulina fijadora de hormonas sexuales). 
hipogonadismo en pacientes con disfunción eréctil aumenta claramente con la edad y en función del nivel sérico establecido como criterio diagnóstico (13). Si se emplea para el diagnóstico los niveles séricos de testosterona libre en vez de la testosterona total, la prevalencia también es mayor, pudiendo aumentar de un $5 \%$ a un $18 \%$ en alguna serie (14).

En cualquier caso, la evaluación inicial de todos los varones con disfunción eréctil debe incluir una determinación de testosterona sérica, ya que ésta permite detectar un número significativo de pacientes que pueden beneficiarse de la terapia androgénica (3). Como mínimo debe evaluarse la testosterona total, aunque según otra corriente de opinión la determinación de testosterona libre o biodisponible también son necesarias ya que son las formas biológicamente activas (14).

\section{Influencia de la testosterona en la fisiopatología de la erección}

La erección es un proceso complejo que precisa de la integridad de las propiedades fibroelásticas del tejido cavernoso y en el que intervienen neurotransmisores, factores endocrinos y agentes vasoactivos (15).

Los estudios en animales de experimentación han aportado múltiples evidencias sobre el efecto modulador de la testosterona sobre la función eréctil:

- Estudios experimentales en ratas han demostrado que la castración altera la ultraestructura del nervio dorsal del pene (16) y de los nervios cavernosos (2). También se ha comprobado que la erección producida por estimulación del área preóptica medial en ratas es testosterona-dependiente (17). Por lo tanto, la testosterona parece regular en el animal de experimentación los mecanismos neurológicos de la erección tanto a nivel central como periférico.

- Se ha demostrado en diversos estudios experimentales que la testosterona regula la expresión y actividad de las distintas isoformas (neuronal y endotelial) de la sintetasa de óxido nítrico (NOS) en el cuerpo cavernoso de la rata $(18,19)$. Es conocido que el óxido nítrico es el mediador de la relajación del músculo liso trabecular y arterial intracavernoso, via activación de la guanilciclasa (Figura 2). En animales castrados, los suplementos de testosterona o dihidrotestosterona (DHT) restablecieron la función eréctil y la expresión de NOS en el cuerpo cavernoso $(18,19)$.

- También se ha demostrado que la testosterona regula la actividad de la fosfodiesterasa 5 (PDE5). La
PDE5 hidroliza el GMP cíclico (cGMP) en 5'GMP a nivel del músculo liso vascular y trabecular. La castración en conejos (20) y de ratas (21) reduce la expresión y actividad de la PDE5, restaurándose las mismas tras la administración de testosterona. Es más, los animales castrados no respondían in vivo a los inhibidores de la PDE5 (21). Por lo tanto, la testosterona regularía tanto el inicio de la erección (mediante la activación de NOS) como su finalización (mediante la activación de la PDE5). Aunque ambas acciones parecen contradictorias, se han interpretado como parte de un mismo proceso de homeostasis (Figura 2) para mantener unos niveles adecuados de enzimas críticos en la fisiología de la erección (2).

- Además del papel en la regulación bioquímica en el proceso fisiológico de la erección, también se han observado cambios estructurales en el tejido cavernoso en conejos castrados, que afectan especialmente a la activación y mantenimiento del mecanismo venooclusivo, ya que se produce una reducción en el tejido muscular liso y un incremento de tejido conectivo, con acúmulos de adipocitos en el espacio subtunical, que contribuyen al fallo de este mecanismo $(22,23)$. Los andrógenos parecen promover la diferenciación de células madre pluripotentes en células de estirpe muscular, inhibiendo su diferenciación en células de estirpe adiposa (23). Por otra parte, estudios en ratas han demostrado que la castración induce apoptosis en células del tejido espongiocavernoso, que puede ser bloqueada mediante administración de testosterona (24).

- En el animal de experimentación, la función eréctil depende de un umbral crítico de testosterona. Se ha demostrado que, en ratas castradas, la función eréctil se correlaciona positiva y significativamente con los niveles séricos de testosterona hasta alcanzar éstos un umbral crítico, por encima del cual la función eréctil es comparable a la de los animales no castrados. Este umbral es bajo, siendo un $10-12 \%$ de las concentraciones fisiológicas de testosterona (25).

En el humano no se han podido establecer evidencias tan firmes sobre la influencia de la testosterona en la función eréctil como a nivel experimental. No obstante, estudios realizados en varones con hipogonadismo severo, sugieren que el deseo sexual, la frecuencia de la actividad sexual y las erecciones espontáneas nocturnas son claramente dependientes de la testosterona, mientras que las erecciones psicógenas, la eyaculación y el orgasmo serían sólo parcialmente dependientes de la testosterona (12).

En el hombre también se ha descrito la existencia de un umbral en los niveles séricos de testoste- 
rona, por debajo del cual se afecta la función sexual. Este umbral varía considerablemente de unos individuos a otros, aunque parece ser consistente para un mismo individuo y podría ser específico para cada parámetro de la función sexual (26).

Aunque la mayoría de los varones jóvenes con hipogonadismo severo presentan disminución del deseo sexual y disfunción eréctil, los estudios epidemiológicos en varones añosos no han podido demostrar de forma consistente una clara correlación entre los niveles séricos de testosterona total con la presencia y la severidad de disfunción eréctil (12). Incluso en algunos estudios en pacientes hipogonádicos añosos se ha detectado una relación de la severidad de la disfunción eréctil con niveles séricos disminuidos de testosterona libre pero no con los niveles séricos de testosterona total $(14,27)$.

Quizás las evidencias más claras sobre la influencia de la testosterona en la fisiología de la erección en humanos se hayan obtenido mediante estudios con doppler color tras inyección intracavernosa de alprostadil. Foresta et al (28) demostraron flujos sistólicos en arterias cavernosas significativamente menores en pacientes con disfunción eréctil e hipogonadismo que en pacientes con disfunción eréctil de causa psicógena. El índice de resistividad en arterias cavernosas se relacionaba muy significativamente con los niveles séricos de testosterona libre (29), sugiriendo que la relación de los niveles bajos de testosterona con el deterioro del mecanismo de la erección vendría determinada por la influencia de la testosterona sobre los mecanismos de alteración del endotelio.

Por otra parte, la relación entre los niveles de testosterona y el deseo sexual, claramente establecida cuando la testosterona alcanza niveles de castración, es menos evidente en los varones mayores con niveles bajos de testosterona. En algunos estudios no se ha encontrado relación entre los niveles del testosterona y deseo sexual $(14,27)$ y la capacidad predictiva de niveles bajos de testosterona a

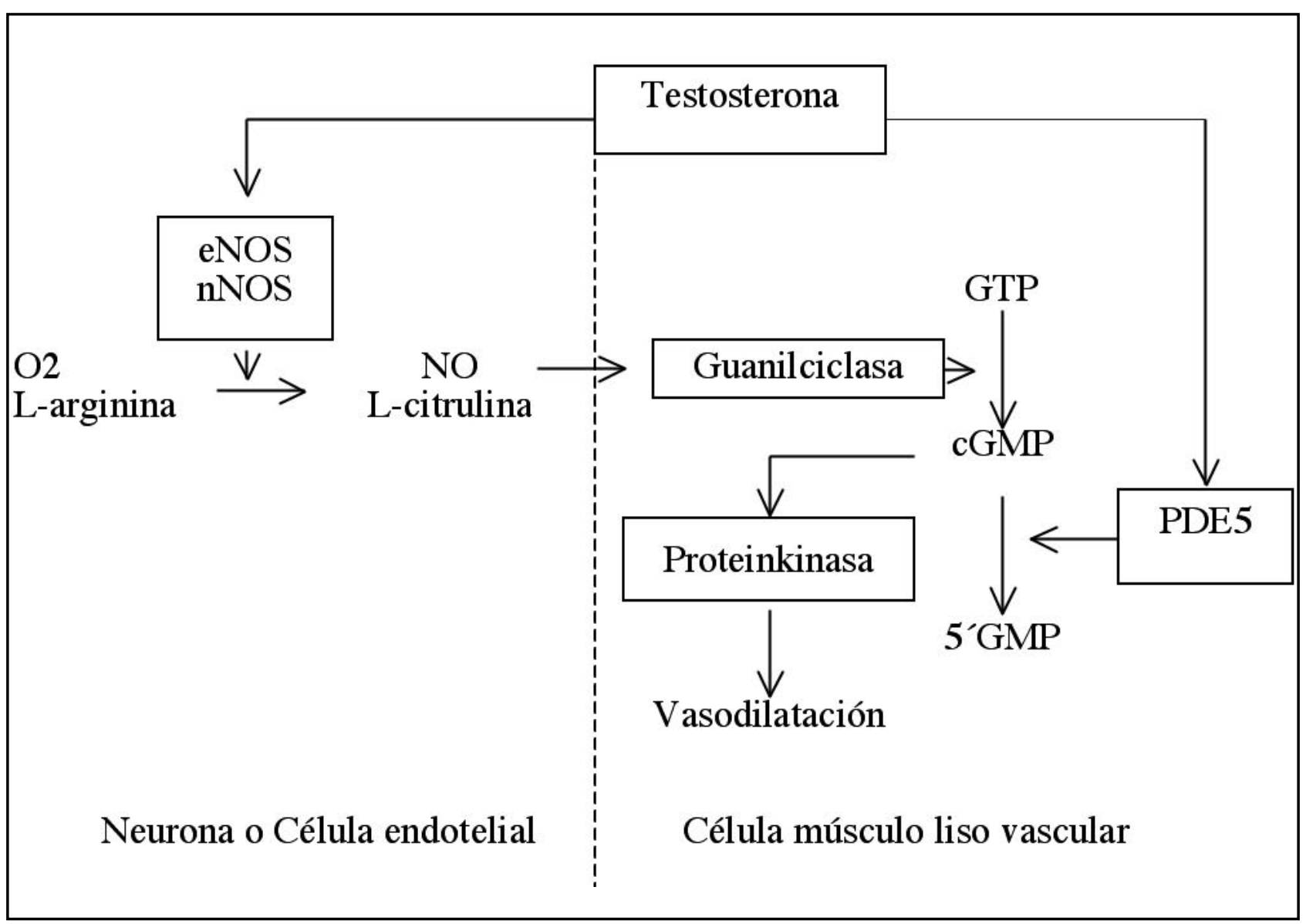

FIGURA 2. Regulación de NOS y PDE5 por la testosterona (adaptado de 2)

leNOS: sintetasa de oxido nítrico endotelial, nNOS: sintetasa de oxido nítrico neuronal, NO: oxido nítrico, cGMP: GMP cíclico, PDE5: Fosfodiesterasa 5) 
partir de la disminución del deseo sexual tiene una baja sensibilidad, especificidad y eficiencia (30) En el mecanismo del deseo sexual están involucrados mecanismos biológicos y psicológicos complejos en los que la testosterona tiene un impacto significativo durante la pubertad (31), pero tendría una influencia más relativa cuando el descenso no es excesivamente marcado, como ocurre en el déficit de testosterona relacionado con el envejecimiento.

\section{Tratamiento de la disfunción eréctil con testosterona}

Los estudios en los que se han evaluado los resultados del tratamiento sustitutivo con testosterona (TST) sobre la función eréctil en varones con niveles bajos de testosterona han proporcionado resultados variables. Desgraciadamente, muchos de los estudios publicados no son controlados con placebo, incluyen a pocos pacientes y no siempre se correlacionan los resultados con los niveles previos de testosterona, lo cual dificulta su interpretación (11).

Jain et al (32) publicaron en 2000 los resultados de un metanálisis que incluía 16 estudios, 5 de ellos controlados con placebo. Las tasas de respuesta al tratamiento en varones hipogonádicos fueron significativamente superiores con TST $(65 \%)$ que con placebo (17\%). Isidori et al (33) publicaron 5 años más tarde otro metanálisis incluyendo 17 ensayos clínicos randomizados en los que se había evaluado los efectos del TST sobre la función sexual en varones hipogonádicos de cualquier edad. Se detectó una mejoría significativa de todos los parámetros de la función sexual en comparación con placebo, siempre que los niveles de testosterona fueran inferiores a 12 $\mathrm{nmol} / \mathrm{L}$. La magnitud del efecto del TST sobre la función eréctil estaba inversamente relacionada con los niveles séricos de testosterona. También se comprobó que la respuesta al TST estaba influida por factores como el tipo de testosterona administrada, la longitud del seguimiento, y la existencia de comorbilidad (diabetes, hipertensión, etc.). Boloña et al (34), en otro metanálisis más reciente, concluyeron que el TST en varones con disfunción sexual se asociaba con una mejoría discreta de la función eréctil y con una mejoría moderada de la libido.

Los metanálisis de Isidori et al (33) y Boloña et al (34) incluyen algunos pacientes jóvenes con hipogonadismo prepuberal, los cuales suelen tener una mejor respuesta al TST. Sin embargo, la eficacia del TST en varones mayores con SDT parece ser menor: en una compilación de 8 estudios observacionales sólo el $36 \%$ de este tipo de pacientes mejoró definitivamente su función eréctil con TST (11). Probablemente esta menor tasa de respuesta al TST sea debi- da a que en una proporción de pacientes coexisten niveles bajos de testosterona con otros factores etiológicos de la disfunción eréctil, siendo en estos casos la disfunción eréctil multifactorial, y no únicamente debida al déficit androgénico. Por ello, en los casos de SDT en los cuales el TST no mejora la disfunción eréctil se ha ensayado el tratamiento combinado con inhibidores de PDE5. Greenstein et al (35), en un estudio no controlado, trataron a 48 pacientes con SDT y disfunción eréctil con gel de testosterona durante 6 meses, mejorando la función eréctil en 31 (65\%). En 17 casos la disfunción eréctil no mejoró, pero sí lo hizo en todos ellos tras asociar sildenafilo al TST durante 3 meses.

La misma estrategia de tratamiento combinado se ha empleado en pacientes con disfunción eréctil en los que fracasa el tratamiento inicial con inhibidores de PDE5. Un 30-40\% de no respondedores a inhibidores de PDE5 presentan niveles disminuidos de testosterona (12). El efecto modulador que parecen ejercer los andrógenos sobre la expresión de PDE5 (20) y la posible necesidad de unos niveles normales de testosterona para que los inhibidores de PDE5 sean eficaces (21), aunque son extremos que no han sido totalmente confirmados en humanos, podrían justificar el tratamiento combinado en estos casos. De hecho, en diversas series se ha observado que un $52-92 \%$ de pacientes hipogonádicos con disfunción eréctil que no respondieron inicialmente al tratamiento con inhibidores de PDE5, sí lo hicieron al asociar testosterona (12).

Dos estudios controlados con placebo parecen confirmar estos hallazgos: Aversa et al (36) trataron a 20 pacientes con disfunción eréctil e hipogonadismo que no respondían a sildenafilo mediante parches de testosterona o de placebo junto con sildenafilo. El grupo tratado con testosterona más sildenafilo experimentó una mejoría significativa tanto en la sintomatología como en el flujo sistólico intracavernoso medido con doppler color en comparación con el grupo tratado con placebo más sildenafilo. Shabsigh et al (37), por su parte, trataron a 75 varones hipogonádicos con disfunción eréctil sin respuesta a sildenafilo con gel de testosterona o de placebo más sildenafilo, observando una mejoría significativa en las puntuaciones del IIEF a las 4 semanas en el grupo tratado con testosterona.

Aunque es necesario un mayor número de estudios controlados incluyendo un mayor número de pacientes, el tratamiento combinado parece ser un recurso adecuado para rescatar pacientes con disfunción eréctil e hipogonadismo que no responden a la monoterapia, ya sea ésta testosterona sóla o inhibidores de PDE5 sólos. 


\section{CONCLUSIONES}

La real prevalencia del SDT es desconocida, pero se estima que entre un $5-15 \%$ de varones con disfunción eréctil presentan niveles séricos disminuidos de testosterona.

Existe una sólida base experimental que demuestra que la testosterona juega un papel fundamental en la fisiología de la erección, tanto a nivel central como periférico. No obstante, las evidencias obtenidas en humanos no son tan firmes, especialmente en los varones mayores con SDT.

La determinación sistemática de testosterona sérica en los pacientes que consultan por disfunción eréctil es altamente recomendable, ya que el TST permite, en una proporción de pacientes, mejorar las erecciones y el deseo sexual. Además, el TST puede mejorar el resto de síntomas del SDT y aumentar la eficacia de los inhibidores de la PDE5 cuando éstos no son eficaces como monoterapia.

\section{BIBLIOGRAFIA y LECTURAS RECOMENDADAS ( ${ }^{*}$ lectura de interés $y^{* *}$ lectura fundamental)}

1. Bancroft J, Wu FC: Changes in erectile responsiveness during androgen replacement therapy. Arch Sex Behav 1983; 12: 59-66.

*2. Traish AM, Goldstein I, Kim NN: Testosterone and erectile function: From basic research to a new clinical paradigm for managing men with androgen insufficiency and erectile dysfunction. Eur Urol 2007; 52:54-70.

**3. Wang C, Nieschlag E, Swerdloff R, Behre HM, Hellstrom WJ, Gooren LJ et al: Investigation, treatment, and monitoring of Late-Onset Hypogonadism in males: ISA, ISSAM, EAU, EAA and ASA recommendations. Eur Urol 2009; 55:12130.

4. Laaksonen DE, Niskanen L, Punnonen K, Nyyssonen K, Tuomainen TP, Valkonen VP et al: Testosterone and sex hormone-binding globulin predict the metabolic syndrome and diabetes in middleage men. Diabetes Care 2004; 27:1036-41.

5. Yassin AA, Saad F, Gooren LJ: Metabolic syndrome, testosterone deficiency and erectile dysfunction never come alone. Andrologia 2008; 40:25964.

6. Pardridge WM. Selective delivery of sex steroid hormones to tissues in vivo by albumin and by sex hormone-binding globulin. Ann NY Acad Sci. 1988; 538:173-92.
7. Vermeulen A, Verdonck L, Kaufman JM: A critical evaluation of simple methods for the estimation of free testosterone in serum. J Clin Endocrinol Metab 1999; 84:3666-72.

8. Martínez Jabaloyas JM, Queipo Zaragozá A, Gil Salom M, Chuan Nuez P: Evaluación de una técnica de inmunoanálisis para la determinación de testosterona libre. Actas Urol Esp 2006; 30: 598-601.

*9. Harman SM, Metter EJ, Tobin JD, Pearson J, Blackman MR; Baltimore Longitudinal Study of Aging. Longitudinal effects of aging on serum total and free testosterone levels in healthy men. Baltimore Longitudinal Study of Aging. J Clin Endocrinol Metab 2001; 86:724-31

10. Martínez Jabaloyas JM, Queipo Zaragozá A, Ferrandis Cortés C, Queipo Zaragozá JA, Gil Salom M, Chuan Nuez P: Cambios en las hormonas sexuales en varones mayores de 50 años. Prevalencia de niveles bajos de testosterona y factores de riesgo. Actas Urol Esp 2008;32:603-610.

11. Mikhail N: Does testosterone have a role in erectile function? Am J Med 2006; 119: 373-382.

12. Buvat J, Bou Jaoudé G: Significance of hypogonadism in erectile dysfunction. World $\mathbf{J}$ Urol 2006; 24:657-67.

13. Köhler TS, Kim J, Feia K, Bodie J, Johnson N, Makhlouf A, Monga M: Prevalence of Androgen Deficiency in Men with Erectile Dysfunction. Urology 2008; 71: 693-7.

*14. Martínez-Jabaloyas JM, Queipo-Zaragozá A, Pastor-Hernández F, Gil-Salom M, Chuan-Nuez P: Testosterone levels in men with erectile dysfunction. BJU Int 2006; 97:1278-83.

15. Traish AM, Park K, Dhir V, Kim NN, Moreland $\mathrm{RB}$, Goldstein I: Effects of castration and androgen replacement on erectile function in a rabbit model. Endocrinology 1999; 140:1861-8.

16. Rogers RS, Graziottin TM, Lin CS, Kan YW, Lue TF: Intracavernosal vascular endothelial growth factor (VEGF) injection and adeno-associated virus-mediated VEGF gene therapy prevent and reverse venogenic erectile dysfunction in rats. Int J Impot Res 2003; 15:26-37.

17. Suzuki N, Sato Y, Hisasue S, Kato R, Suzuki K, Tsukamoto T: Effect of testosterone on intracavernous pressure elicited with electrical stimulation of the medial preoptic area and cavernous nerve in male rats. J Androl 2007; 28:218-22.

18. Garban H, Marquez D, Cai L, Rajfer J, Gonzalez-Cadavid NF: Restoration of normal adult penile erectile response in aged rats by long-term treatment with androgens. Biol Reprod 1995; 53:1365-72.

19. Marin R, Escrig A, Abreu P, Mas M: Androgendependent nitric oxide release in rat penis correlates with levels of constitutive nitric oxide synthase isoenzymes. Biol Reprod 1999; 61:1012-6. 
20. Morelli A, Filippi S, Mancina R, Luconi M, Vignozzi L, Marini $\mathrm{M}$, et al: Androgens regulate phosphodiesterase type 5 expression and functional activity in corpora cavernosa. Endocrinology 2004; 145:2253-63.

*21. Zhang XH, Morelli A, Luconic M, Vignozzi L, Filippi S, Marini M, et al: Testosterone regulates PDE5 expression and in vivo responsiveness to tadalafil in rat corpus cavernosum. Eur Urol 2005; 47:409-16.

22. Traish AM, Park K, Dhir V, Kim NN, Moreland $\mathrm{RB}$, Goldstein I: Effects of castration and androgen replacement on erectile function in a rabbit model. Endocrinology 1999; 140:1861-8

23. Traish AM, Toselli P, Jeong SJ, Kim NN: Adipocyte accumulation in penile corpus cavernosum of the orchiectomized rabbit: a potential mechanism for veno-occlusive dysfunction in androgen deficiency. J Androl 2005; 26:242-8.

24. Podlasek CA: Sonic hedgehog, apoptosis, and the penis. J Sex Med 2009; 6 Suppl 3:334-9.

*25. Armagan A, Kim NN, Goldstein I, Traish AM: Dose-response relationship between testosterone and erectile function: evidence for the existence of a critical threshold. J Androl 2006; 27:517-26.

26. Kelleher S, Conway AJ, Handelsman DJ: Blood testosterone threshold for androgen deficiency symptoms. J Clin Endocrinol Metab 2004; 89:3813-7.

27. Ahn HS, Park CM, Lee SW: The clinical relevance of sex hormone levels and sexual activity in the ageing male. BJU Int 2002; 89:526-30.

*28. Foresta C, Caretta N, Rossato M, Garolla A, Ferlin A: Role of androgens in erectile function. $\mathrm{J}$ Urol. 2004; 171:2358-62.

**29. Aversa A, Isidori AM, De Martino MU, Caprio M, Fabbrini E, Rocchietti-March M, et al: Androgens and penile erection: evidence for a direct relationship between free testosterone and cavernous va- sodilation in men with erectile dysfunction. Clin Endocrinol (Oxf). $2000 ; 53: 517-22$.

30. Buvat J, Lemaire A: Endocrine screening in 1,022 men with erectile dysfunction: clinical significance and cost-effective strategy. J Urol 1997; 158:1764-7.

31. Meuleman EJ, van Lankveld JJ: Hypoactive sexual desire disorder: an underestimated condition in men. BJU Int 2005; 95:291-6.

*32. Jain P, Rademaker AW, McVary KT: Testosterone supplementation for erectile dysfunction: results of a meta-analysis. J Urol 2000; 164:371-5.

*33. Isidori AM, Gianetta E, Gianfrilli D, Greco EA, Bonifacio V, Aversa A et al: Effects on testosterone on sexual function in men: results of a meta-analysis. Clin Endocrinol (Oxf) 2005; 63:381-94.

*34. Boloña ER, Uraga MV, Haddad RM, Tracz MJ, Sideras K, Kennedy CC et a: Testosterone use in men with sexual dysfunction: A systematic review and meta-analysis of randomized placebo-controlled trials. Mayo Clin Proc 2007; 82:20-28.

*35. Greenstein A, Mabjeesh NJ, Sofer M, Kaver I, Matzkin H, Chen J: Does sildenafil combined with testosterone gel improve erectile dysfunction in hypogonadal men in whom testosterone supplement therapy alone failed? J Urol 2005; 173:530-532.

36. Aversa A, Isidori AM, Spera G, Lenzi A, Fabbri A: Androgens improve cavernous vasodilation and response to sildenafil in patients with erectile dysfunction. Clin Endocrinol (Oxf) 2003; 58:632-8.

**37. Shabsigh R, Kaufman JM, Steidle C, Padma-Nathan H: Randomized study of testosterone gel as adjunctive therapy to sildenafil in hypogonadal men with erectile dysfunction who do not respond to sildenafil alone. J Urol 2004; 172:658-63. 